\title{
Disinfection of Spacecraft Potable Water Systems by Photocatalytic Oxidation Using UV-A Light Emitting Diodes
}

\author{
Michele N. Birmele ${ }^{1}$ \\ Engineering Services Contract, Team QNA, Kennedy Space Center, Florida, 32899 \\ Jeremy A. O'Neal ${ }^{2}$ \\ NASA USRP Intern, Kennedy Space Center, Florida, 32899 \\ and \\ Michael S. Roberts ${ }^{3}$ \\ Engineering Services Contract, Team QNA, Kennedy Space Center, Florida, 32899
}

Ultraviolet (UV) light has long been used in terrestrial water treatment systems for photodisinfection and the removal of organic compounds by several processes including photoadsorption, photolysis, and photocatalytic oxidation/reduction. Despite its effectiveness for water treatment, UV has not been explored for spacecraft applications because of concerns about the safety and reliability of mercury-containing UV lamps. However, recent advances in ultraviolet light emitting diodes (UV LEDs) have enabled the utilization of nanomaterials that possess the appropriate optical properties for the manufacture of LEDs capable of producing monochromatic light at germicidal wavelengths. This report describes the testing of a commercial-off-the-shelf, high power Nichia UV-A LED $\left(250 \mathrm{~mW} \lambda_{365 \mathrm{~nm}}\right)$ for the excitation of titanium dioxide as a point-of-use (POU) disinfection device in a potable water system. The combination of an immobilized, high surface area photocatalyst with a UV-A LED is promising for potable water system disinfection since toxic chemicals and resupply requirements are reduced. No additional consumables like chemical biocides, absorption columns, or filters are required to disinfect and/or remove potentially toxic disinfectants from the potable water prior to use. Experiments were conducted in a static test stand consisting of a polypropylene microtiter plate containing $3 \mathrm{~mm}$ glass balls coated with titanium dioxide. Wells filled with water were exposed to ultraviolet light from an actively-cooled UV-A LED positioned above each well and inoculated with six individual challenge microorganisms recovered from the International Space Station (ISS): Burkholderia cepacia, Cupriavidus metallidurans, Methylobacterium fujisawaense, Pseudomonas aeruginosa, Sphingomonas paucimobilis and Wautersia basilensis. Exposure to the Nichia UV-A LED with photocatalytic oxidation resulted in a complete ( $>7-\log )$ reduction of each challenge bacteria population in $<\mathbf{1 8 0}$ minutes of contact time. With continued advances in the design and manufacture of UV-A LEDs and semi-conducting photocatalysts, LED activated photochemical process technology promises to extend its application to spacecraft environmental systems.

\section{Nomenclature}

$\lambda \quad=$ wavelength

$<\quad=$ less than

$>\quad=$ greater than

${ }^{\circ} \mathrm{C} \quad=$ degrees celsius

${ }^{1}$ Research Scientist, Engineering Services Contract, Team QNA, ESC-3.

${ }^{2}$ NASA USRP Intern from the University of Florida, Biological Engineering Dept., Gainesville, Florida.

${ }^{3}$ Ground Research Team Lead, Engineering Services Contract, Team QNA, ESC-3.

1

American Institute of Aeronautics and Astronautics 


$\begin{array}{ll}\text { Amp } & =\text { ampere } \\ \text { AO } & =\text { acridine orange } \\ \text { AODC } & =\text { acridine orange direct counts } \\ \text { CFU } & =\text { colony forming units } \\ \text { COTS } & =\text { commercial of the shelf } \\ \text { DNA } & =\text { deoxyribonucleic acid } \\ \text { eV } & =\text { electron volts } \\ \text { g } & =\text { grams } \\ \text { HOMO } & =\text { highest unoccupied molecular orbital } \\ \text { HPC } & =\text { heterotrophic plate count } \\ \text { ISS } & =\text { International Space Station } \\ \text { LED } & =\text { light emitting diode } \\ \text { L } & =\text { liters } \\ \text { log } & =\text { logarithm } \\ \text { LP } & =\text { low pressure } \\ \text { LUMO } & =\text { lowest unoccupied molecular orbital } \\ \mathrm{mL} & =\text { milliliters } \\ \mathrm{mW} & =\text { milliwatts } \\ \mathrm{nm} & =\text { nanometer } \\ \mathrm{PCO} & =\text { photocatalytic oxidation } \\ \mathrm{POU} & =\text { point-of-use } \\ \mathrm{TiO} & \\ \mathrm{TOC} & =\text { titanium dioxide } \\ \mathrm{UV} & =\text { total organic carbon } \\ \mathrm{UV}-\mathrm{A} & =\text { ultraviolet } \\ \mathrm{V} & =\text { volt } \\ \mathrm{W} & =\text { watts } \\ & \end{array}$

\section{Introduction and Background}

$\mathrm{O}$ ne of the major concerns for maintaining potable water in spacecrafts for both the crew and the flight hardware is the possibility of microbial contamination and the advancement of biofilm formation. Current disinfection methods require the addition of chemical biocides that reduce bacterial and fungal populations through ionic interference of biochemical pathways and then point-of-use (POU) filters are required to lower the end-point concentration of these chemical biocides to levels that are safe for human consumption. Iodine and ionic silver (silver fluoride and/or silver nitrate) are typically used on the Shuttle and International Space Station (ISS) to disinfect water systems. There are several disadvantages to these methods which generate a need for advances in antimicrobial technology. These chemicals can have relatively low human toxicity thresholds and cannot be implemented in high dosages. They also lose efficacy over time which requires multiple repeat dosages and increases the demands on valuable and limited crew time. For example, iodine has high initial Total Organic Carbon (TOC), reported unfavorable flavor, and requires point-of-use (POU) carbon filtering. In addition, iodine and ionic silver cannot be combined since a chemical reaction causes the iodine and ionic silver to precipitate out of solution as silver iodine. This occurance greatly reduces antimicrobial properties to

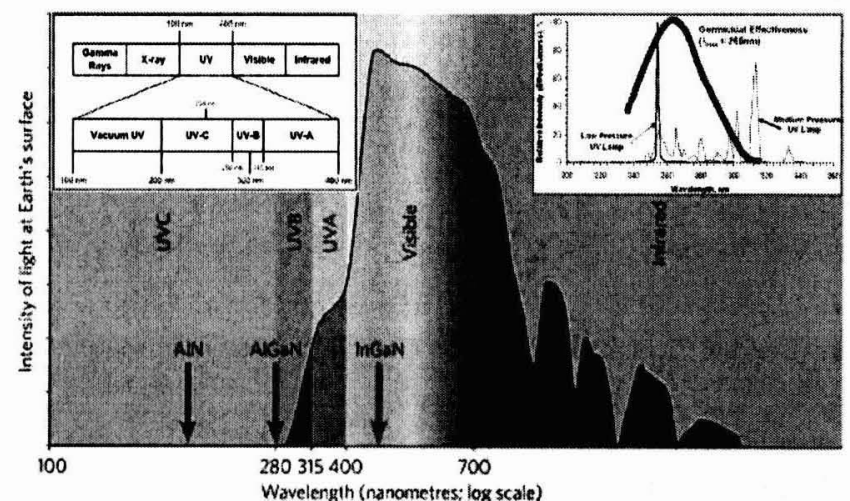

Figure 1. Electromagnetic Spectrum of Ultraviolet Light. 
the point where the chemicals are considered inert for this purpose.

Among the effort to implement advanced microbial control, technologies to collect, store, recycle, and disinfect water for use/reuse as drinking and hygiene water have been identified as critical needs for potable water systems in spacecraft. In particular, the volume, mass, and energy constraints necessary to supply and maintain potable water for crew use will require the recovery, processing, and storage of water from multiple sources including urine, hygiene water, humidity condensate, and/or water derived from in situ resources. ${ }^{9}$ Treatment processes must be capable of producing and maintaining potable and hygiene water supplies that meet NASA Human System Integration Requirements (HSIR) for the next generation of vehicles. ${ }^{9,11}$ Additionally, these processes should achieve high levels of mass closure that minimize power and volume requirements for long duration missions., 11

Due to the aforementioned reasons, various experiments have been performed to test the viability of UV-LEDs as a low power disinfection method for potable water systems for both terrestrial and space applications. ${ }^{1} \mathrm{UV}$ light breaks down DNA, preventing replication and damaging proteins by forming dimers between nucleic acids and hindering transcription. ${ }^{13}$ This does not immediately kill the microbe but can render it incapable of replication and therefore prevents it from being pathogenic. ${ }^{5}$ UV light comes in a wide spectrum from $100-400 \mathrm{~nm}$ that is divided into 4 components (UV-vacuum, A, B, and C) as shown in Fig. $1 .{ }^{4}$ The ultraviolet range chosen for this experiment was UV-A because of the stability and durability of the LEDs being produced and their availability as a commercialoff-the-shelf (COTS) product. UV-A (median $\lambda=365 \mathrm{~nm}$ ) has also shown mutagenic capabilities to damage DNA resulting in effects similar, although decreased in capacity, to UV-C (median $\lambda=265 \mathrm{~nm}$ ). ${ }^{12}$ The microbial inactivation efficacy of UV-A light for disinfection of water is augmented by addition of photocatalysts (e.g., titanium dioxide) that can be oxidized by UV-A to produce free oxygen radicals that in turn damage microorganisms by attacking proteins in cell membranes. ${ }^{3}$

UV-LEDs were chosen over mercury UV lamps due to the toxicity of the mercury contained in them. The power consumption in LEDs is also much better than mercury UV lamps, current UV-LEDs use between 250-310 mW while mercury UV lamps use wattages of $60 \mathrm{~W}$ and higher. ${ }^{7}$ Mercury lamps are relatively efficient at generating UV light in the germicidal spectrum (i.e., peak output at $253.7 \mathrm{~nm}$ for LP lamps, which is near the DNA maximum germicidal absorbance of $265 \mathrm{~nm}$ ) but suffer from a high power penalty. ${ }^{11}$ Mercury UV lamps would also not pass an engineering safety review due to acute toxicity concerns. ${ }^{10}$ UV-A LEDs use solid state electronics, don't contain mercury, and new designs are able to emit light near $365 \mathrm{~nm}$ which is above the optimal of $265 \mathrm{~nm}$, so the addition of a photocatalyst was needed to boost antimicrobial efficacy as previously mentioned. ${ }^{\text {? }}$

Photocatalysts are materials that have the ability to absorb UV and/or visible light and transfer electrons to neighboring molecules. The electron transfer pathway contains multiple steps. Initially the photocatalyst absorbs light which excites the ground state electrons from the lowest unoccupied molecular orbital (LUMO) into the highest unoccupied molecular orbital (HOMO). The electron desires to be at the lowest energy state; therefore the electron will decay energy levels back to the ground state. When the neighboring molecule has a LUMO that is higher than that of the catalyst, the electron will transfer to the neighboring molecule before decaying back to the original ground state LUMO of the catalyst. The donation of the electron to a neighboring molecule can then be used to catalyze a chemical reaction, generate power, or disrupt biochemical reactions. The band gap of the photocatalyst between the LUMO and HOMO determines the efficiency of the material to donate electrons. The higher the band gap, the more efficient the photocatalyst is at energy generation because more transfer steps can occur before the electron reaches its original ground state. Titanium dioxide is a well know photocatalyst with a band gap of around $3.0 \mathrm{eV}$. Doping and crystal structure changes can tailor the band gap between 2.8 and $3.2 \mathrm{eV}$. In this study, we examined both rutile and anatase crystal structures of titanium dioxide with a band gap of around $3.0 \mathrm{eV}$ to promote electron transfer between the material and the bacteria within solution to generate photocatalytic oxidation
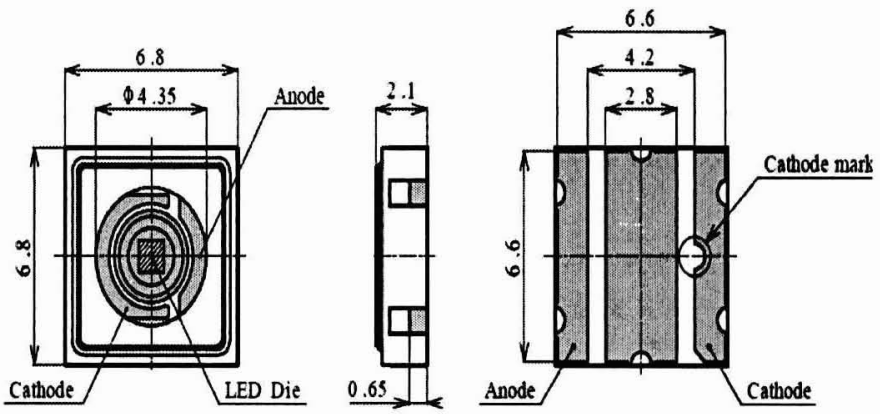

Figure 2. UV-A High Power I-LED; 365 nm; 250 mW Model NCSU033A (T) Specifications.

(PCO). Photocatalytic oxidation creates free radicals which, in water, can create hydroxyl radicals. These hydroxyl radicals can oxidize organic compounds and add to the affect of UV-A light. ${ }^{8}$ 


\section{Materials and Methods}

A. UV-A LEDs and Titanium Dioxide

The specifications for the UV-A LEDs used in this experiment are described in Fig. 2 and Fig. 3. The UVA LEDs used a current of $300 \mathrm{~mA}$ and voltage of $24 \mathrm{~V}$. The fans used to cool the LEDs were set at $90 \mathrm{~mA}$ and $12 \mathrm{~V}$. Three types of titanium dioxide (SigmaAldrich, St. Louis, MO) were used in this experiment. Solutions of rutile titanium dioxide were synthesized at concentrations of $40 \mathrm{~g} / \mathrm{L}$ and $4 \mathrm{~g} / \mathrm{L}$ in ethanol (Sigma). The solutions were deposited onto the test wells of separate 96 well plates using an evaporation method. The anatase crystal phase of titanium dioxide used was synthesized into beads via sol-gel evaporation. 20 anatase beads were placed at the bottom of each test well in a 96 well plate.
(2) Initial Electrical'Optical Characteristics

$\left(\mathrm{Ts}=25^{\circ} \mathrm{C}\right)$

\begin{tabular}{|c|c|c|c|c|c|c|c|}
\hline \multicolumn{2}{|l|}{ Item } & Symbol & Condition & Min. & Typ. & Max. & Unit \\
\hline \multirow{3}{*}{ Forward Vollage } & Rank H & \multirow{3}{*}{$V_{F}$} & \multirow{3}{*}{$\mid \mathrm{F}=500[\mathrm{~mA}]$} & 4.0 & . & 4.4 & \multirow{3}{*}{ v } \\
\hline & Rank M & & & 3.6 & - & 4.0 & \\
\hline & \begin{tabular}{|l|} 
Rank L. \\
\end{tabular} & & & 3.2 & . & 3.6 & \\
\hline Peal Wavelength & Rank Ua & $\lambda . P$ & $\lfloor\mathrm{f}=500[\mathrm{~mA}]$ & 360 & $(365)$ & 370 & $\mathrm{~nm}$ \\
\hline \multicolumn{2}{|l|}{ Spectrum Half Width } & $\Delta i$ & {$[\mathrm{~F}=500[\mathrm{~mA}]$} & . & (9) &. & $\mathrm{nm}$ \\
\hline \multirow{3}{*}{ Radiant Flux } & Rank P7 & \multirow{3}{*}{$\phi$} & \multirow{3}{*}{$\mathrm{F}=500[\mathrm{~mA}]$} & 270 &. & 310 & \multirow{3}{*}{$\mathrm{mW}$} \\
\hline & Rank P6 & & & 230 & . & 270 & \\
\hline & Rank P5 & & & 190 & . & 230 & \\
\hline
\end{tabular}

* $T_{\mathrm{s}}$ : The solder teunerature of products (Please refer to the drawing. 090609826792.)

$*$ Fonvard Voltage Measurement allowance is $\approx 3 \%$.

* Peak Wavelength Measurement allowance is $=3 \mathrm{~nm}$.

* Radiant Flux Measurement allowance is $\pm 10 \%$

Figure 3. UV-A High Power I-LED; $365 \mathrm{~nm} ; 250 \mathrm{~mW}$ Model NCSU033A (T) Specifications.

\section{B. Lifecycle Performance}

To measure the performance of the UV-A LEDs, the light intensity was measured on an Optronics Laboratory OL 754 Spectroradiometer. Measurements were taken before and after exposure to measure the light intensity degradation over time.

\section{Challenge Organisms}

The six bacterial organisms chosen for this experiment were found to be the most likely contaminates to potable water and environmental control life support systems in space due to some of their unique abilities to resist antimicrobials. The challenge organisms include: Burkholderia cepacia (ATCC 25416), Cupriavidus metallidurans (ATCC 43123), Methylobacterium fujisawaense (ATCC 43884), Pseudomonas aeruginosa (ATCC 10145), Sphingomonas paucimobilis (ATCC 29837), and Wautersia basilensis (ISS strain 073130023-1).

\section{Static Experimentation}

Two test stands, designated board A and board B, according to the particular Nichia UV-A LEDs, were used. An inoculum was prepared by growing each of the test bacterial cells individually in tryptic soy broth (BD, Difco, Franklin Lakes, NJ) and resuspending them in sterile deionized water. An inoculum of $1 \times 10^{7}$ cells $/ \mathrm{mL}$ was added in triplicate to a 96 well plate, $2 \mathrm{~mL}$ per well. They were then exposed to each permutation of the treatments while being sampled every 15-30 minutes of exposure time for up to three hours. Control wells were also prepared the same way, but were not exposed to a treatment. Another control test was held in a separate 96 well plate and kept away from the treatment plate to avoid UV exposure. These tests were performed to all of the challenge organisms separately. Duplicate tests were conducted to compare the efficacy of each board. A temperature monitoring experiment was also conducted using a thermocouple while taking measurements of the temperature change over 90 minutes in 15 minutes intervals for both boards. All tests were conducted in a controlled environmental chamber set at 20 degrees Celsius, $50 \%$ humidity, and $400 \mathrm{ppm} \mathrm{CO}_{2}$.

\section{E. Microbial Analysis Methods}

A spectrophotometer was employed to (1) measure rapid cellular concentration at $590 \mathrm{~nm}$ and (2) determine the initial concentration of the inoculum. Heterotrophic plate counts (HPC) on R2 agar (R2A) were used to calculate $\mathrm{CFU} / \mathrm{mL}$. Only cells which are able to reproduce can grow on this medium. Acridine orange direct counts (AODC)

4 
fixed in $2 \%$ formalin were used to fluorescent stain live and dead cells. ${ }^{2,6}$ The stained cells were then analyzed and enumerated using a Zeiss Axioskop epi-fluorescent microscope.

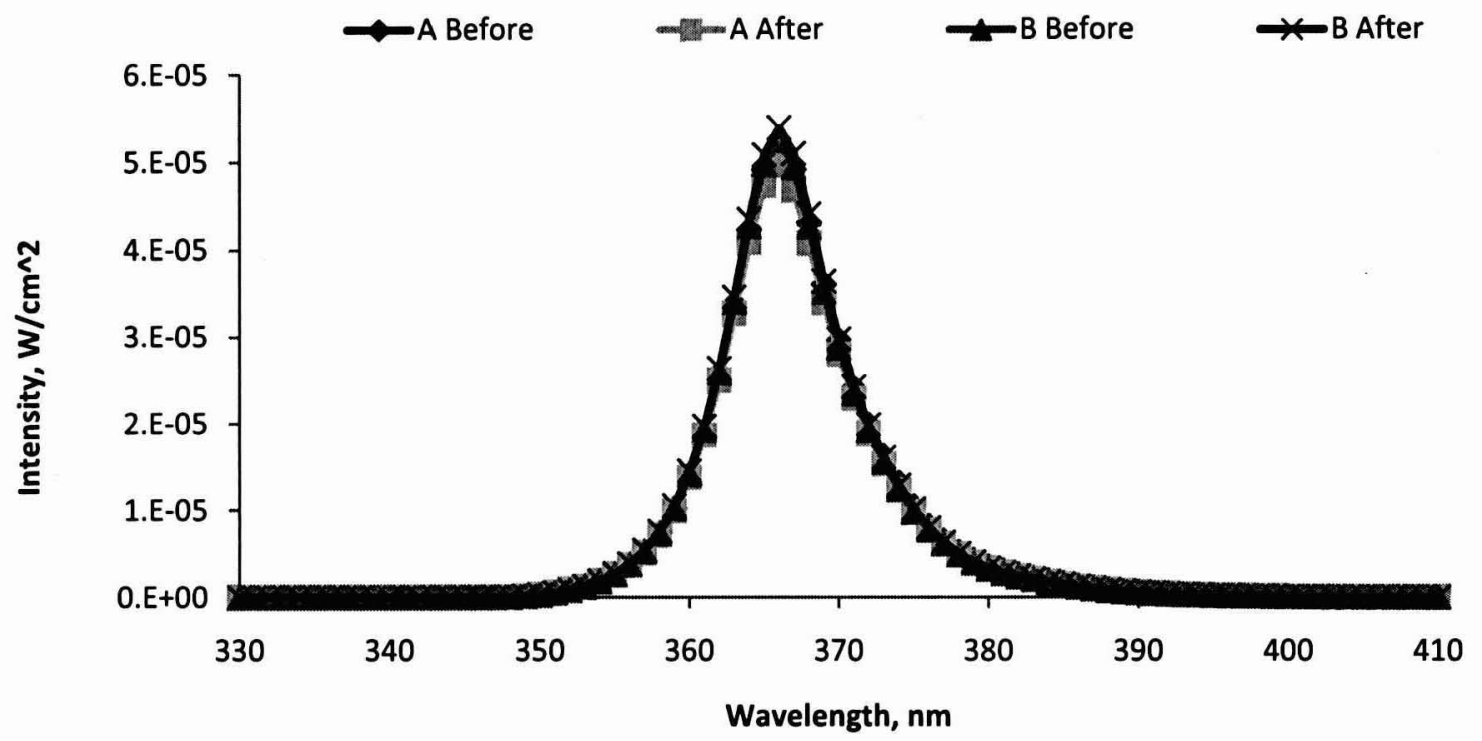

Figure 4. UV-A LED electro-optical performance for Boards A and B.

\section{Results}

\section{A. UV-A LED Performance}

The performance of the UV-A LEDs is shown in Fig. 4. According to this data, there were negligable variations between board A and board B, and also between each individual LED after 100 hours of operation. The average decrease was less than $0.01 \%$ between the initial and the endpoint scans.

\section{B. Temperature Monitoring}

The temperature of the water increased from $22^{\circ} \mathrm{C}$ to $25^{\circ} \mathrm{C}$ and then leveled out at the $25^{\circ} \mathrm{C}$ after 30 minutes for the remainder of testing for both of the boards. This change in temperature should not have contributed to any loss in cellular viability.

\section{AODC Results}

Very little decrease was found before and after exposure to the UV-A LEDs with and without the addition of titanium dioxide according to the AODCs (Table 1). This data supports the theory that the cells were still present in the sample but were no longer culturable. Since the cell membranes were not disrupted, the bacteria would continue to metabolize until cell death, but would be unable to replicate.

\section{UV-A HPC Results}

UV-A LED exposure effectively disinfected all of the challenge organisms to a non-detectable level within 90 minutes except for Sphingomonas paucimobilis and Methylobacterium fujisawaense which were only reduced about $1-\log$. The 4 challenge organisms that were reduced by UV-A alone, indicated a 7+-log reduction in bacteria populations within the experimental time frame. The UV-A LED with anatase titanium dioxide beads performed similarly to the control UV-A LED exposed samples for the six challenge organisms by killing all measureable bacteria within the inoculums (Fig. 5). In addition, the anatase $\mathrm{TiO}_{2} / \mathrm{UV}-\mathrm{A}$ LEDs had a slightly greater affect on Sphingomonas paucimobilis and Methylobacterium fujisawaense. The test with UV-A and $40 \mathrm{~g} / \mathrm{L}$ of rutile titanium dioxide only yielded a 1-2 log reduction over 120 minutes due to particulates blocking the cellular absorbance of the UV-A. The UV-A with $4 \mathrm{~g} / \mathrm{L}$ of rutile titanium dioxide, yielded a 7-log reduction as well but it took 120 minutes

5

American Institute of Aeronautics and Astronautics 
again due to particulate shielding the cells. Exposure to only titanium dioxide yielded a $0.06 \log$ reduction in three hours, which was a very minimal impact.

Table 1. AODC and HPC Results ( $n=6)$.

Before exposure $\quad$ After exposure

\begin{tabular}{ccccc}
\hline $\log (10)$ & AODC (cells/mL) & HPC (CFU/mL) & AODC (cells $/ \mathrm{mL})$ & HPC (CFU/mL) \\
\hline $\begin{array}{c}\text { Burkholderia } \\
\text { cepacia }\end{array}$ & $6.83 \pm 0.02$ & $6.92 \pm 0.82$ & $6.79 \pm 0.04$ & $0.00 \pm 0.00$ \\
\hline
\end{tabular}

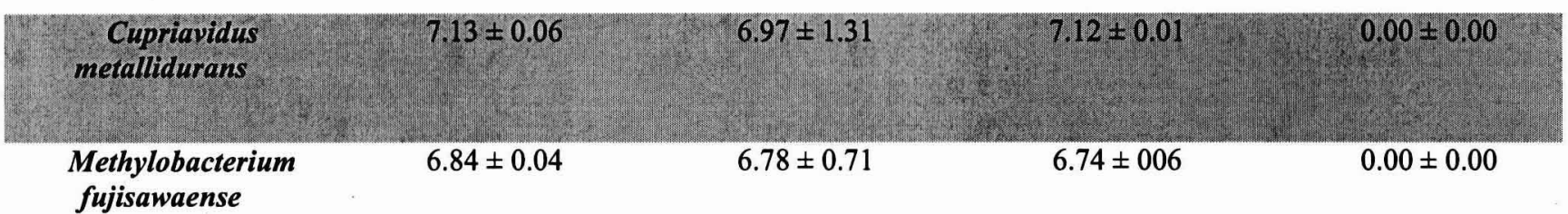
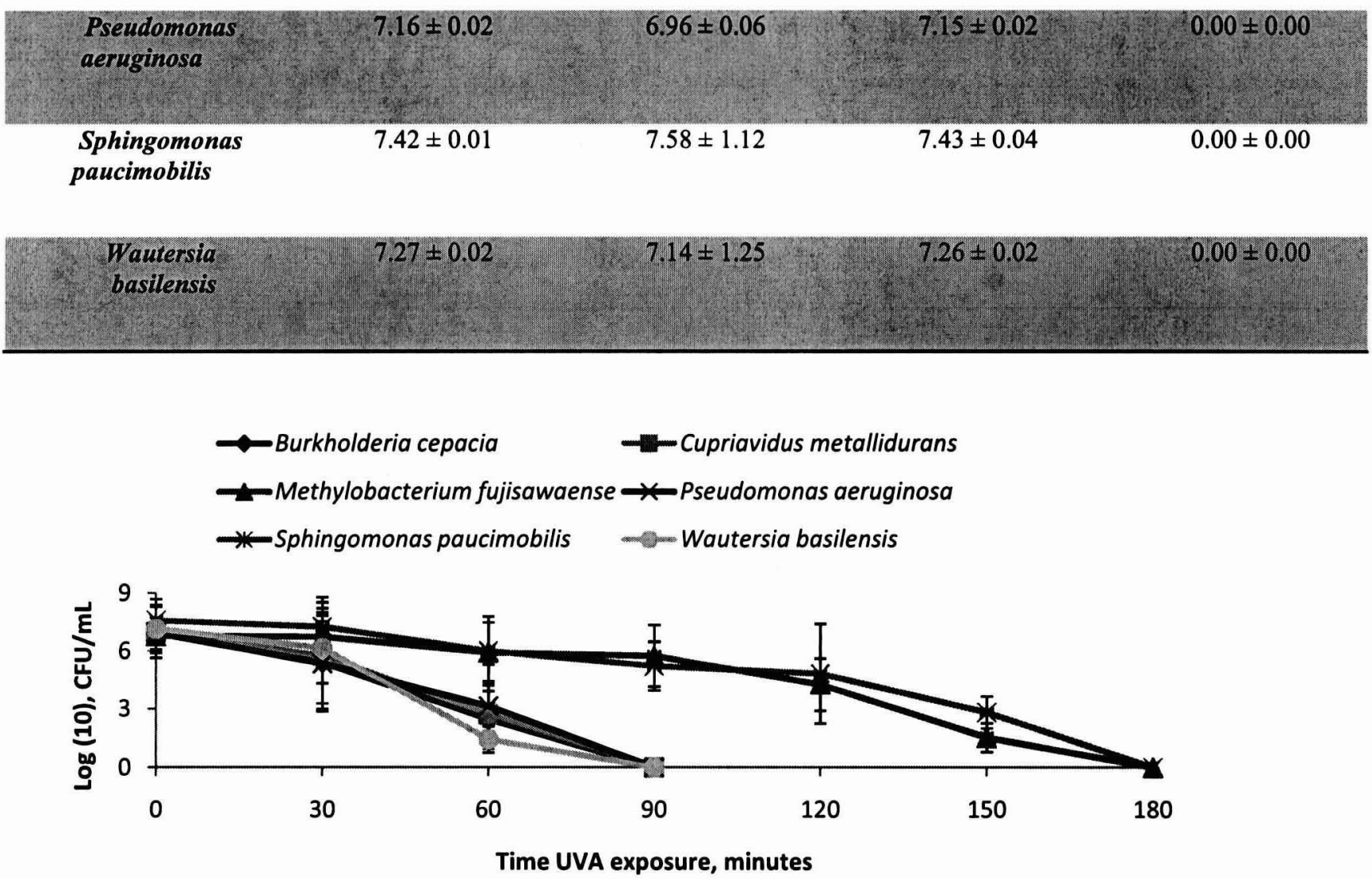

Figure 5. UVA HPC Results.

$H P C$ results of all six organisms exposed to UV-A with anatase phase titania( $n=3)$.

6

American Institute of Aeronautics and Astronautics 


\section{Conclusions}

The data from the spectroradiometer showed that there is low variability in intensity between LEDs and between boards. Therefore, in theory, all UV-A LEDs and both boards would have similar efficacy during experimentation. Temperature monitoring for this test stand showed that the UV-A LEDs alone do not produce enough heat to kill the challenge organisms. The AODC was performed to determine the mechanism of cellular degradation. The data showed that the bacteria were not being impacted by cellular membrane rupture caused by the UV-A or the photocatalyst. Rather it confirmed that the UV-A and titanium dioxide are disrupting replication through DNA degradation. Disrupting cellular replication works as efficiently as rupturing or killing the cells because they are rendered non-pathogenic.

The HPCs revealed that UV-A or UV-A plus titanium dioxide was a very effective disinfection method for static water systems. Out of the six challenge bacteria, the UV-A with anatase titanium dioxide beads was able to reduce Sphingomonas paucimobilis and Methylobacterium fujisawaense more effectively than UV-A alone. This indicated that the titanium dioxide contributed to the disinfection. The rutile titanium dioxide did not perform as well as the anatase beads because the rutile film detached from the walls of the well, floated to the surface of the solution, and blocked the UV-A from penetrating the top of the water. It can also be concluded from these experiments that titanium dioxide without a source of UV light is not effective at disinfection. Furthermore, tests will need to be performed to accurately monitor the formation of oxygen reactive species.

\section{Acknowledgments}

The authors wish to express their sincere appreciation to Megan Morford, John Catechis, Griffin Lunn, and the NASA Life Science Services Contract (NAS10-02001) (Dynamac Corp.) for research support. The authors also wish to thank Dr. Andrew C. Schuerger (University of Florida) for the use of an OL-754 spectroradiometer.

\section{References}

\footnotetext{
${ }^{1}$ Birmele, M., McCoy, L., Soler, R., and Roberts, M.S. Ultraviolet Light Emitting Diodes for Disinfection of Spacecraft Potable Water Systems. ICES 2009 09ICES-0083 / 2009-01-2508.

${ }^{2}$ Bloem, J., "Fluorescent staining of microbes for total direct count," In Molecular Microbial Ecology Manual, Vol. 8, Kluwer Academic Publishers, Netherlands, 1995, pp. 1-12.

${ }^{3}$ Giese, N., and Darby, J., "Sensitivity of microorganisms to different wavelengths of UV light: implication on modeling of medium pressure UV systems," Water Researc, Vol. 34, No. 16, 2000, pp. 4007-4013.

${ }^{4}$ Hamamoto, A., Mori M., Takahashi, A., Nakaya, Y., and Kinouchi, Y., "New water disinfection system using UV-A lightemitting diodes," Journal of Applied Microbiology, Vol. 103, 2007, pp. 2291-2298.

${ }^{5}$ Hijnen, W.A.M., Beerendonk, E.F., Medema, G.J., "Inactivation credit of UV radiation for viruses, bacteria and protozoan (oo)cysts in water: a review," Water Research, Vol. 40, No. 1, 2006, pp. 3-22.

${ }^{6}$ Hobbie, J.E., Daley, R.J., and Jasper, S., "Use of Nucleopore filters for counting bacteria by fluorescence microscopy," Applied Environmental Microbioogy, Vol. 33, 1977, pp. 1225-1228.

${ }^{7}$ Khan, A., “A bug-beating diode," Nature, Vol. 441, 2006, pp. 299.

${ }^{8}$ Liu, B., Wen, L., Zhao, X., "The study of photocatalysis under ultraviolet + visible two-beam light irradiation using undoped nano-titanium dioxide," Materials Chemistry and Physics, Vol. 112, 2008, pp. 35-40.

9 NASA, "Constellation Program Human-Systems Integration Requirements," CxP 70024, Baseline, 2007. (NASA-CxP70024)

${ }^{10}$ NASA, “Man-Systems Integration Standards," Revision B, July 1995. (NASA-STD-3000)

${ }^{11}$ Naunovic, Z., Lim, S., and Blatchley III, E. R., "Investigation of microbial inactivation efficiency of a UV disinfection system employing an excimer lamp," Water Research, Vol. 42, 2008, pp. 4838-4846.
}

7

American Institute of Aeronautics and Astronautics 
12 Runger, T., and Kappes U. P., "Mechanisms of mutation formation with long-wave ultraviolet light (UV-A)," Photodermatology, Photoimmunology \& Photomedicine, Vol. 24, 2007, pp. 2-10.

${ }^{13}$ Vermeulen, N., Keeler, W.J., Nandakumar, K., and Leung, K.T., "The bactericidal effect of ultraviolet and visible light on Escherichia coli," Biotechnology and Bioengineering, Vol. 99, 2008, pp. 550-556. 